\title{
OBTAINING REAL PARTS OF SCATTERING AMPLITUDES DIRECTLY FROM CROSS SECTION DATA USING DERIVATIVE ANALYTICITY RELATIONS
}

\author{
J.B. BRONZAN ${ }^{*}$ \\ National Accelerator Laboratory, Batavia, Illinois 60510, USA \\ and \\ G.L. KANE and U.P. SUKHATME \\ Physics Department, University of Michigan, Ann Arbor, Michigan 48104, USA
}

Received 13 March 1974

\begin{abstract}
We show that one can obtain real parts of scattering amplitudes by knowing the imaginary parts at only nearby energies. This is accomplished by re-casting the dispersion integral into an equivalent form which we will call a "derivative analyticity relation". Predictions are given for forward amplitudes where $\sigma_{\mathrm{T}}$ is measured: $\mathrm{pp}, \overline{\mathrm{p}} \mathrm{p}, \mathrm{K}^{ \pm} \mathrm{p}$, $\pi^{ \pm} \mathrm{p}, \mathrm{p}$. We deduce the real part of the elastic pp amplitude away from the forward direction at ISR energies.
\end{abstract}

Dispersion relations give the real part of an amplitude in terms of an integral over its imaginary part. Because the connection is nonlocal, the real part at high energy appears to depend upon the behavior of the imaginary part at all energies. We will show that at high energy this cumbersome nonlocal connection can be replaced by a quasilocal relation in which the real part is given in terms of the imaginary part and its derivatives at the same energy. Comparing our "derivative analyticity relation" with available data for forward scattering, we will demonstrate that it is easy to apply and very useful at least down to resonance energies. We will also apply the quasilocal relation to the relatively untouched subject of the phase of amplitudes with $t \approx 0$.

We derive our relation for an even (crossing symmetric) amplitude $f_{+}$(with poles and subtraction constant removed for simplicity). This amplitude normalized so that $\left.s \sigma_{\mathrm{T}}^{+}=\operatorname{Im} f_{+}(s, 0)\right]$ satisfies the subtracted dispersion relation

$\operatorname{Re} f_{+}(s, t)=\frac{2 s^{2}}{\pi} \mathrm{P} \int_{s_{0}}^{\infty} \frac{\mathrm{d} s^{\prime}}{s^{\prime}\left(s^{\prime 2}-s^{2}\right)} \operatorname{Im} f_{+}\left(s^{\prime}, t\right)$.

Integrate by parts and replace $s\left(s^{\prime}\right)$ by $\mathrm{e}^{\xi}\left(\mathrm{e}^{\xi^{\prime}}\right)$,

F Permanent address: Physics Department, Rutgers University, New Brunswick, New Jersey 08903.

㭋 Research supported in part by the U.S. Atomic Energy Commission.

$$
\begin{aligned}
& \operatorname{Re} f_{+}(s, t)=\frac{s}{\pi} \int_{\ln s_{0}}^{\infty} \mathrm{d} \xi^{\prime} s^{\prime \alpha-1}\left[\ln \operatorname{coth} \frac{1}{2}\left|\xi-\xi^{\prime}\right|\right] \\
& \times\left(\alpha-1+\frac{\mathrm{d}}{\mathrm{d} \xi^{\prime}}\right) \operatorname{lm} f_{+}\left(s^{\prime}, t\right) / s^{\prime} \alpha .
\end{aligned}
$$

The kernel in brackets is positive, peaked at $\xi=\xi^{\prime}$ and decreases like $2 \exp \left(-\left|\xi^{\prime}-\xi\right|\right)$ for $\left|\xi^{\prime}-\xi\right|$ large. At this point we expand $\operatorname{Im} f_{+}\left(s^{\prime}, t\right) / s^{\prime} \alpha$ in powers of $\xi^{\prime}-\xi$, and exchange order of integration and summation; since energy is large we also extend the lower limit to $-\infty$. After some manipulation, we obtain the derivative analyticity relation $[1,2]$

$$
\begin{aligned}
\operatorname{Re} f_{+}(s, t)=s^{\alpha} \tan \left[\frac{1}{2} \pi\left(\alpha-1+\frac{\mathrm{d}}{\mathrm{d} \ln s}\right)\right] \operatorname{Im} f_{+}(s, t) / s^{\alpha} \\
=\tan \left[\frac{1}{2} \pi(\alpha-1)\right] \operatorname{Im} f_{+}(s, t) \\
\quad+\frac{1}{2} s^{\alpha} \pi \sec ^{2}\left[\frac{1}{2}(\alpha-1)\right] \frac{\mathrm{d}}{\mathrm{d} \ln s} \operatorname{Im} f_{+}(s, t) / s^{\alpha}+\ldots
\end{aligned}
$$

The analogous relation for odd (crossing antisymmetric) amplitudes is

$$
\begin{aligned}
\operatorname{Re} f_{-}(s, t)=s^{\alpha}\left[\tan \frac{1}{2} \pi\left(\alpha+\frac{\alpha}{\alpha \ln s}\right)\right] \operatorname{Im} f_{-}(s, t) / s^{\alpha} \\
=\tan \left(\frac{1}{2} \pi \alpha\right) \operatorname{Im} f_{-}(s, t) \\
\quad+\frac{1}{2} s^{\alpha} \pi \sec ^{2}\left(\frac{1}{2} \pi \alpha\right) \frac{\alpha}{\alpha \ln s}\left(\operatorname{Im} f_{-}(s, t) / s^{\alpha}\right)+\ldots
\end{aligned}
$$



(4).

A number of points can be made about eqs. (3) and

(1) A careful selection of the parameter $\alpha$ is unnecessary. For example, suppose we choose $\alpha=1$, which is appropriate for the Pomeranchukon at $t=0$. Then if $\operatorname{Im} f_{+} \sim s^{\bar{\alpha}}$, the leading term in eq. (3) is $22 \%$ low when $\bar{\alpha}=1 / 2$. This point can be put slightly differently: when eq. (3) (with $\alpha=1$ ) is applied to forward scat. tering, one can expect accuracy of a few percent even when secondary Regge exchanges are appreciable.

(2) Eqs. (3) and (4) are technically asymptotic (high energy) relations. Errors can be expected at low energy because the lower limit has been extended in eq. (2), because of other thresholds, and because of pole terms.

(3) Only the first two terms in eqs. (3) and (4) can be determined by contemporary data. Above the resonance region the first two terms yield good numerical accuracy except where the graph of $\operatorname{Im} f_{+}$against lns is very curved.

(4) In view of eq. (3), the asymptotic behavior of $\operatorname{Im} f_{+}$affects $\operatorname{Re} f_{+}$primarily by changing the local value of $\mathrm{d} f_{+} / \mathrm{d} \ln s$. Since this derivative can be obtained directly from the data, one can learn nothing about total cross sections at energies much above some point $s$ by a measurement of $\operatorname{Re} f_{+}$at $s$.

In order to apply eq. (4) for $t \pm 0$, we must face three problems. First crossing symmetric and antisymmetric amplitudes cannot be formed simply by combining cross section data. We bypass this problem by working at high energy where the Pomeranchukon, and hence, the even amplitude, dominates. This assumption presumably fails at diffraction minima, and our formulas will not be valid there.

The second problem is that the only quantity measured at $t \pm 0$ is $\mathrm{do} / \mathrm{d} t$, and to apply eq. (4) we must know the imaginary part. However, at ISR energies the diffraction peak is no longer shrinking much at large $|t|$, even though the minima are slowly moving. This suggests $\alpha \approx 1$ and dominance by the imaginary part except at dips. We therefore have $\left(\operatorname{Im} f_{\lambda}\right)^{2} \approx\left|f_{\lambda}\right|^{2}$ away from dips. $\lambda$ is the collection of all helicity indices.

The third problem is that spin cannot be ignored at $t \pm 0$. However, suppose we define $\rho_{\lambda}(s)$ to be the fractional contribution of helicity channel $\lambda$ to $\mathrm{d} \sigma / \mathrm{d} t$. Using only the leading term in eq. (3) we have
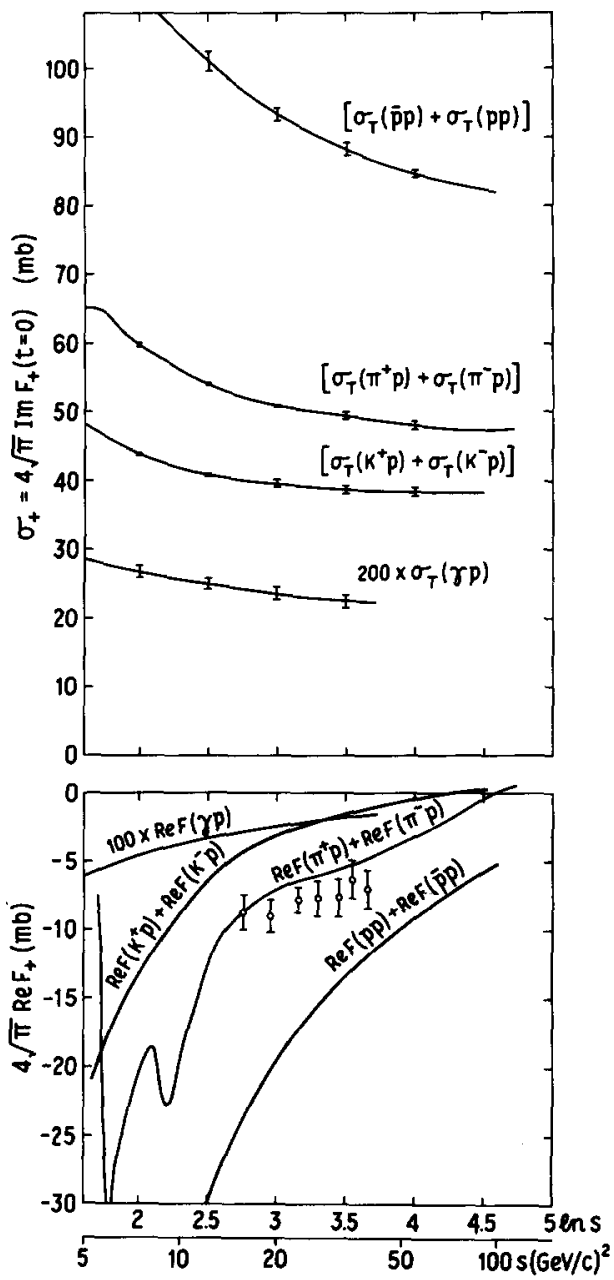

Fig. 1. The imaginary parts of the even signature amplitudes at $t=0$ (i.e., $\sigma \mathrm{T}$ ) are shown plotted as functions of $\ln s$. The even signature real parts for the various processes are shown calculated from the imaginary parts using the derivative analyticity relation $\operatorname{Re} F_{+}=(\pi / 2) d o_{T} / d$ lns [eq. (3)]. Note that in the region $s>50(\mathrm{GeV} / s)^{2}$ where the $\pi \mathrm{N}$ and $\mathrm{KN}$ even signature cross sections are essentially flat, one must have the even signature $\pi \mathrm{N}$ and $\mathrm{KN}$ real parts essentially zero. Available data points are shown for the $\pi \mathrm{N}$ even signature real part.

$\frac{1}{4} \pi \frac{\mathrm{d}}{\mathrm{d} \ln s} \ln \frac{\mathrm{d} o}{\mathrm{~d} t} \approx \sum_{\lambda} \rho_{\lambda} \operatorname{Re} f_{\lambda} / \operatorname{Im} f_{\lambda}$.

This formula gives the one piece of information about the phase of helicity amplitudes that can be extracted from $\mathrm{d} \sigma / \mathrm{d} t$.

The results of a number of applications are given in the figures. In all the figures we have used an amplitude $F$ related to $f$ by $4 \sqrt{\pi} F=f / s$. At $t=0$ wherever 


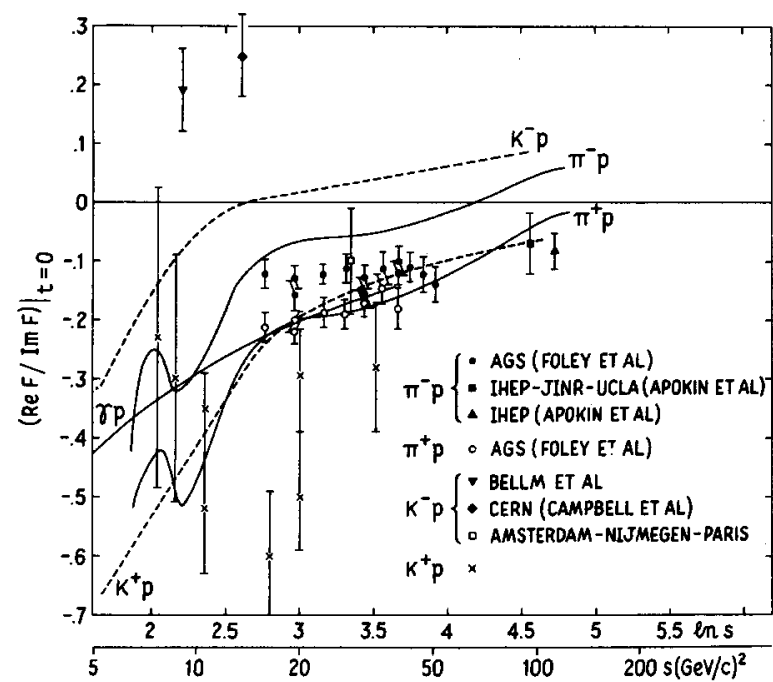

Fig. 2. Predictions for real parts at $t=0$ for separate reactions, obtained by taking odd signature real parts due to a fitted power law fall off for $\Delta \sigma \mathrm{T}$ and even signature real parts from fig. 1 [eq. (6)]. Note that the even signature real parts are approximately between those for the separate reactions since $\operatorname{Re}\left(X^{ \pm} p\right)=\operatorname{Re}($ even signature $) \pm \operatorname{Re}$ (odd signature).

high energy cross sections are measured we have calculated the real part of the even amplitude implied by the energy dependence of total cross sections $[3,4]$. The results are shown in fig. 1. Data is available for $\pi^{ \pm} p$ and the agreement, while reasonable, could be better.

To get $\operatorname{Re} F$ for the separate reactions we need the odd signature contribution also (except for $\gamma \mathrm{p}$ which is purely even signature). We calculated $\operatorname{Re} F_{-}$from eq. (4) by using the data directly and differentiating with respect to $\ln s$, and also by fitting $\Delta \sigma_{\mathrm{T}}$ with a power law $\Delta \sigma_{\mathrm{T}} \equiv \sigma_{\mathrm{T}}^{-} \equiv \sigma_{\mathrm{T}}\left(\mathrm{X}^{-} \mathrm{p}\right)-\sigma_{\mathrm{T}}\left(\mathrm{X}^{+} \mathrm{p}\right)=C p_{\mathrm{lab}}^{-(1-\alpha)}$; the results are qualitatively the same. Our predictions are shown in fig. 2 . In all cases the real parts were computed from formulas

$$
\begin{aligned}
& \operatorname{Re} F\left(\mathrm{X}^{ \pm} \mathrm{p}\right)=\frac{1}{2}\left[\operatorname{Re} F_{+} \pm \operatorname{Re} F_{-}\right] \\
& \approx \frac{1}{2}\left[\frac{1}{2} \pi \frac{\mathrm{d} \sigma_{\mathrm{T}}^{+}}{\mathrm{d} \ln s} \mp \tan \left(\frac{1}{2} \pi \alpha\right) \sigma_{\mathrm{T}}^{-}\right. \\
& \left.\left.\quad+\frac{1}{2} \pi s^{\alpha-1} \sec ^{2}\left(\frac{1}{2} \pi \alpha\right) \frac{\mathrm{d}}{\mathrm{d} \ln s}\left(\frac{\sigma_{\mathrm{T}}^{-}}{s^{\alpha-1}}\right)\right)\right],
\end{aligned}
$$

where $\sigma_{\mathrm{T}}^{ \pm} \equiv \sigma_{\mathrm{T}}\left(\mathrm{X}^{-} \mathrm{p}\right) \pm \sigma_{\mathrm{T}}\left(\mathrm{X}^{+} \mathrm{p}\right)$, with $\alpha=0.68$ for $\pi \mathrm{N}, \alpha=0.44$ for $\mathrm{KN}$ and $\alpha=0.39$ for $\mathrm{NN}$. For even signature, where the errors on $\sigma_{\mathrm{T}}$ are small, we expect

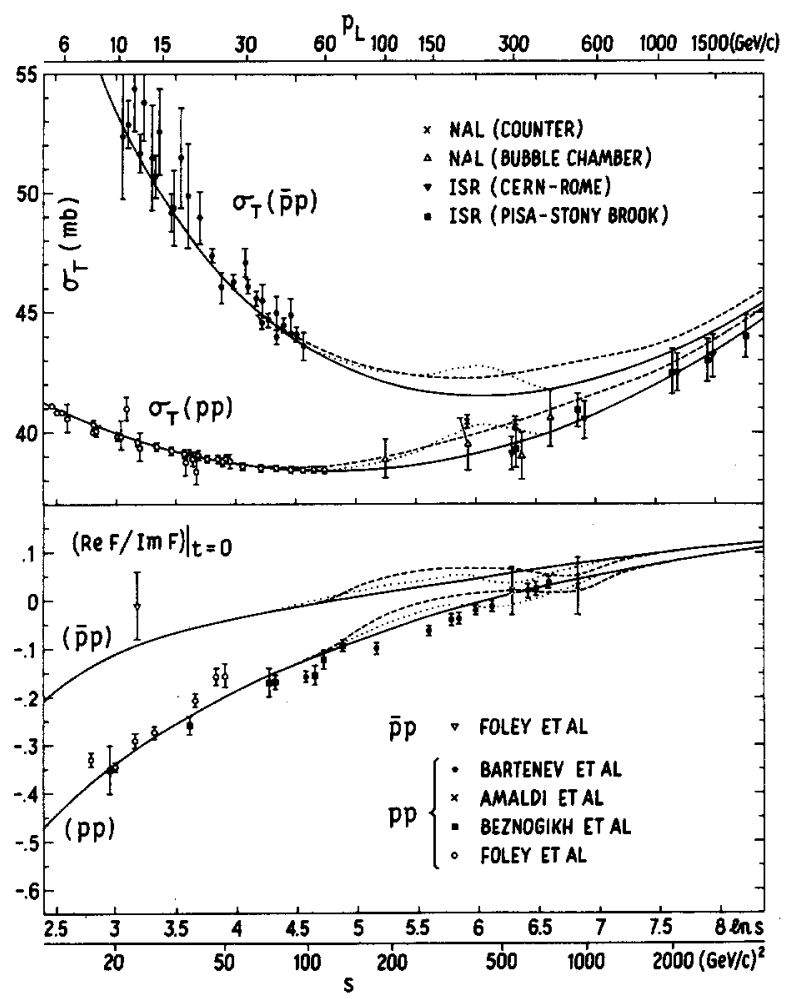

Fig. 3. $\sigma_{\mathrm{T}}$ and $t=0$ real parts as in the previous figures, extended to higher energies for $\mathrm{NN}$ reactions, assuming $\sigma_{\mathrm{T}}(\overline{\mathrm{p} p})$ $-\sigma_{\mathrm{T}}(\mathrm{pp})$ continues to fall at higher energies as it does up to $60 \mathrm{GeV} / c$. The solid lines are $\ln ^{2} s$ smooth curves through the ISR $\sigma_{\mathrm{T}}$ data, with associated real parts calculated from eq. (9) (with $\alpha=0.39$ ). The dashed and dotted $\sigma \mathrm{T}$ lines are instead drawn through the recent NAL $\sigma \mathrm{T}$ data [3], and the dashed and dotted $\operatorname{Re} F$ lines show the resulting real part given our assumptions.

rather small errors on $\operatorname{Re} F_{+}$, say $10-20 \%$. Odd signature is not so well determined because $\Delta \sigma_{\mathrm{T}}$ is not well measured.

For $\pi^{-} p$ the agreement with the available data is not good. The separation between the $\pi^{ \pm} p$ curves is just to the odd signature, and is large because the data for $\Delta \sigma_{\mathrm{T}}$ falls slowly with energy; it is not possible to have $\Delta \sigma_{\mathrm{T}}$ be of significant size and fall slowly and also have equal real parts for $\pi^{ \pm} p$. Which one of the $\pi^{ \pm} p$ curves agrees with data is determined by the even signature real part, whereas the separation depends on the odd. As it stands, the $\sigma_{\mathrm{T}}$ data, the higher energy real part data, and the derivative analyticity relations are not internally consistent, so one or more of them will change. Similar remarks holf for $K^{ \pm} \mathbf{p}$. 


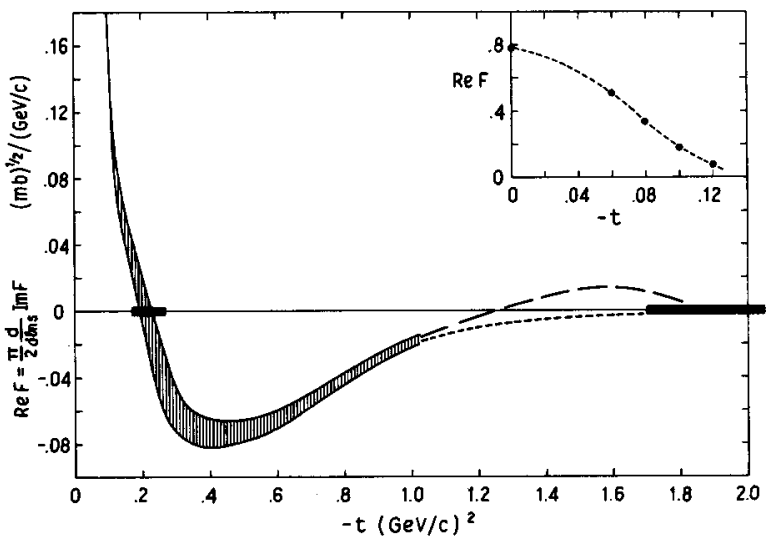

Fig. 4. This shows the real part of the elastic amplitude for $t \leqslant 0$ at $s$ is $2000(\mathrm{GeV} / c)^{2}$ (i.e., the absolute phase of the Pomeranchukon) calculated from $\mathrm{d} \operatorname{Im} F / \mathrm{d} \ln s$ [eq. (3)]. For $-t \leqslant 0.15(\mathrm{GeV} / c)^{2}$ and $0.4 \leqslant-t \leqslant 1(\mathrm{GeV} / c)^{2}$ the result can be deduced from the data [8] (with an uncertainity roughly indicated by the band). The box near $-t=0.2(\mathrm{GeV} / c)^{2}$ shows the $t$ range where the real part has a zero (i.e., the imaginary part is not changing with energy). We have assumed that the cross section is dominated by $\operatorname{Im} F$, with spin effects negligible for $-t \leqslant 1(\mathrm{GeV} / c)^{2}$. At the secondary maximum, the cross section appears to be again essentially energy independent; so if $\operatorname{Im} F$ dominates there then $\operatorname{Re} F$ has another zero there. The dashed lines from $-t \approx 1(\mathrm{GeV} / c)^{2}$ out show two logically possible ways of getting to a zero near $-t \approx 2(\mathrm{GeV} / c)^{2}$.

For $\gamma p$ scattering, we have retained an additional contribution $(4 \pi / \nu)\left(-\alpha / m_{\mathrm{p}}\right),(\nu=$ laboratory energy of the photon) which corresponds to the classical Thomson limit. The resulting real parts are in good agreement with previous dispersion relation calculations [5].

In fig. 3 we extrapolate the NN case to higher energies because of the current interest, in spite of the absence of measured $\sigma_{\mathrm{T}}(\overline{\mathrm{p}} \mathrm{p})$. We assume that $\sigma_{\mathrm{T}}(\overline{\mathrm{p}} \mathrm{p})$ $-\sigma_{\mathrm{T}}(\mathrm{pp})$ continues to fall with energy as observed at Serpukhov. The total cross section data is shown, with some typical smooth curves, through the ISR data and/or through the recent NAL data in the region where there is some conflict. The agreement is less good for the curve through the NAL $\sigma_{T}$ points since it must rise more quickly to get from the low Serpukhov data to the higher points at 200 and $300 \mathrm{GeV} / c$ [6].

The good agreement of $\operatorname{Re} F$ calculated from eq. (6) with the data down to a few $\mathrm{GeV} / c$ for the pp case where the measured $\operatorname{Re} F$ should be most reliable is a useful confirmation that nothing has been ignored in the theory [7].

Finally, we shown in fig. 4 the real part of the ISR elastic non-flip amplitude as a function of $t$ [8]. We should have plotted the averaged ratio of eq. (5). However, to facilitate interpretation we have neglected spin effects and lower lying contributions, assuming that above $s \approx 1500(\mathrm{GeV} / c)^{2}, \mathrm{~d} \sigma / \mathrm{d} t$ is dominated by the imaginary part of the helicity non-flip amplitude (except in the dip region). Two dashed lines in the figure show the logically possible ways to go between $-t \approx 1$ and the secondary maximum; the more positive one has an extra zero in $\operatorname{Re} F$ in the dip region [9].

Note that $\operatorname{Re} F$ will decrease a little faster than Im. $F$ with $-t$ since it has a zero, so the Coulomb interference measurements which are in fact away from $t=0$ will have a small correction; the actual $\operatorname{Re} F$ will be a little larger than the measured one, by perhaps two percent.

The determination of the phase of the Pomeranchukon (via derivative analyticity relations) over a range of $t$ adds a new tool in the analysis of hadron interactions [10].

We have benefited from discussions with F. Hayot, F. Henry, J.D. Jackson and M. Longo.

\section{References}

[1] J.B. Bronzan, Argonne Symposium on the Pomeron, ANL/HEP-7327 (1973) p. 33.

[2] The derivation of the leading term in eq. (3) from the Regge representation was previously known to N.V. Gribov and A.A. Migdal, Yad, Fiz. 8 (1968) 1002 [Sov. J. Nucl. Phys. 8 (1969) 583.]

[3] The total cross section data comes from various compilations of the LBL Particle Data Group and the CERN High Energy Reactions Analysis Group. We also have $\sigma \mathrm{T}$ (pp) data from ISR, NAL bubble chamber measurements, and the recent NAL counter measurements of H.R. Gustafson, L.W. Joner, M.J. Longo and B. Cork, Univ. of Michigan preprint UM HE 73-22 (1973). Phys. Lett., to be published. $\sigma_{T}(\gamma p)$ is taken from D.O. Caldwell et al., Phys. Rev. D7 (1973) 1362.

[4] References to presently available real part data can be found in U. Amaldi, Rapporteur's talk at the Aix-enProvence Conf., September 1973. The recent $\operatorname{Re} F(\mathrm{pp})$ NAL data is taken from

V. Bartenev et al., Phys. Rev. Lett. 31 (1973) 1367.

[5] M. Damashek and F.J. Gilman, Phys. Rev. DS (1970) 1319. 
[6] A report based on our preliminary results (with somewhat improved calculations) is given by J.D. Jackson in his Scottish summer schools lectures, LBL-2079 (1973) p. 39.

[7] Our results are also in good agreement with tradional dispersion relation calculations. See, for example, D.W. Joynson and W. Von Schlippe, Westfield College preprint (1973);

C. Bourrely and J. Fischer, CERN preprint TH-1652 (1973).

[8] We plotted the ISR $\mathrm{d} \sigma / \mathrm{d} t$ data versus at two energies $[\sqrt{s}=30.8$ and $53.2 \mathrm{GeV} / c]$ on the same graph; such a graph is also given by Amaldi [4]. Actually, we used ISR data obtained from $\mathrm{H}$. Miettinen, in which normalizations were carefully treated. The derivative can then be read off directly from the graph at each $t$ value.

[9] Several people have predicted the shape shown in fig. 4. See G.L. Kane, Phys. Lett. 40B (1972) 363 and V.P. Sukhatme and J.N. Ng, University of Washington preprint RLO-1388-652 (1973) to be published in Nucl. Phys. B.

[10] Other recent studies of $\operatorname{Re} F$ at $t \neq 0$ are by P. Kroll, CERN preprint (1973) and

G. Hohler and H.P. Jakob, Karlsruhe preprint (1973). 